\title{
INDUSTRIALIZACIÓN DEL FRUTO DE MOLLE
}

\author{
Raúl Paredes Medina ${ }^{4}$, Walter Flores Ponce de León ${ }^{2}$, \\ María Jordán Ledesma ${ }^{3}$
}

\author{
RESUMEN
}

Se realizó el estudio de la industrialización del fruto de molle a nivel de laboratorio. Logramos obtener chicha de aroma agradable y sabor semidulce, bebida refrescante y medicinal (antidiarreica, recomendable para combatir el mal del cólera), además se elaboró vinagre de aroma característico con $5,0 \%$ de acidez y 2,70 de $\mathrm{pH}$; alcohol etílico del 90,4\% de pureza y aceite esencial con 0,70108 de densidad y 1,4755 de índice de refracción, con buen contenido de ésteres y tocoferoles, de olor agradable semejante al mango y maracuyá.

Los productos obtenidos, fueron analizados según normas ITINTEC con resultados satisfactorios.

\section{A B S TRA C T}

A study of the industrialization of fruit molle was made at laboratory level. We obtained chicha of an agreeable aroma and a semi-sweet flavor, a refreshing and medicinal beverage (to cure diarrhoea, recomended to combat cholera). Moreover vinegar of characteristic aroma with 5,0\% acidity and $\mathrm{pH}$ of 2,70; ethylic alcohol of $90,4 \%$ purity, and esenctial oil with a density of 0,70108 and index refraction of 1,4755 including tocopherols and esters with an agreeable aroma similar to mango and passion fruit, were produced.

The obtained products were analysed according to ITINTEC standards with satisfactory results.

\section{INTRODUCCIÓN}

El molle de nombre científico "Schinus molle", es un árbol resinoso de la familia Anacardiaceae, nativo del área tropical del Perú, Bolivia, Argentina y México. Su hábitat se encuentra en lugares de clima seco, cálido o subcálido.

La parte valiosa de esta planta, son sus frutos drupáceos de color rosado intenso. Referente a su industrialización, es escasa la información bibliográfica, sólo se tienen referencias sobre la elaboración de chicha de manera artesanal por pobladores nativos de la sierra central del pais.

En el presente trabajo, demostramos la factibilidad de la industrialización del fruto de molle, pudiendo ser una alternativa para una pequeña empresa con la elaboración higiénica y aceptable de chicha de molle para consumo humano, del mismo modo la elaboración de vinagre, alcohol etílico y aceite esencial; cuyas obtenciones no requieren de insumos ni materiales o equipos costosos.

\section{MATERIALES Y MÉTODOS}

\section{MATERIALES}

\section{Materia Prima e Insumos}

Frutos maduros y frescos de molle, azúcar rubia, levadura seca, vinagre sin pasteurizar (bacterias acéticas).

\footnotetext{
Magister en Bioquímica.

Ingeniero Químico.

Ingeniero en Industrias Alimentarias.
} 


\section{Reactivos}

Óxido de calcio, hexano, sulfato de sodio anhidro etanol al $95 \%$, fenolftaleína, solución etalónica de hidróxido de potasio, ácido clorhídrico $0,5 \mathrm{~N}$, solución de hidróxido de sodio $0,5 \mathrm{~N}$.

\section{Equipos y Materiales}

Cámara de secado, balanza analítica, cocina eléctrica, equipode destilación, molinomanual, equipo Sohxlet, refrigerador, refractómetro, equipo extractor de aceite esencial, picnómetro, $\mathrm{pH}$ metro, fermentadores, alcoholímetro y materiales de laboratorio de uso común.

\section{MÉTODOS}

\section{Elaboración de Chicha}

Losfrutosmaduros demolle, seleccionados, limpios y secos se sometieron a dilución con agua potable para luego ser percolados a $80^{\circ} \mathrm{C}$ por 30 minutos, se filtró para eliminar los residuos sedimentados. Se prepararon dos muestras y según su grado Brix, a una se corrigió el mosto añadiendo azúcar rubia. Adicionando levadura a las muestras se sometieron a fermentación; la muestra que no requirió corregir mosto, por 7 dias a $20^{\circ} \mathrm{C}$ y otra por 5 días a $22^{\circ} \mathrm{C}$. Se descubaron para separar la fracción grosera de la levadura asentada durante la fermentación y se los filtró al vacío para completar la eliminación de los sedimentos. Finalmente se pasteurizaron a $75^{\circ} \mathrm{C}$ por 15 minutos.

\section{Elaboración de Vinagre}

Los frutos maduros de molle frescos, seleccionados y limpios fueron secados a $60^{\circ} \mathrm{C}$ por 60 minutos, se percolaron con agua potable caliente a $85^{\circ} \mathrm{C}$ por 15 minutos, se filtró para eliminar los frutos y residuos sedimentados. Para lograr las mejores condiciones, se corrigió el mosto añadiendo azúcar y adicionando levadura se sometió a fermentación alcohólica a $22^{\circ} \mathrm{C}$ por 10 días, al cabo de este tiempo se realizó el descube y se pasteurizó a $75^{\circ} \mathrm{C}$ por 15 minutos para detener la fermentación. Añadiendo vinagre fuerte como iniciador se sometió a fermentación acética por siete semanas controlando diariamente su acidez hasta lograr un vinagre adecuado que se filtró y pasteurizó a $65^{\circ} \mathrm{C}$ por 15 minutos para matar las bacterias acidificantes y detener la fermentación.
Flujograma 1. Elaboración de Chicha de Molle.

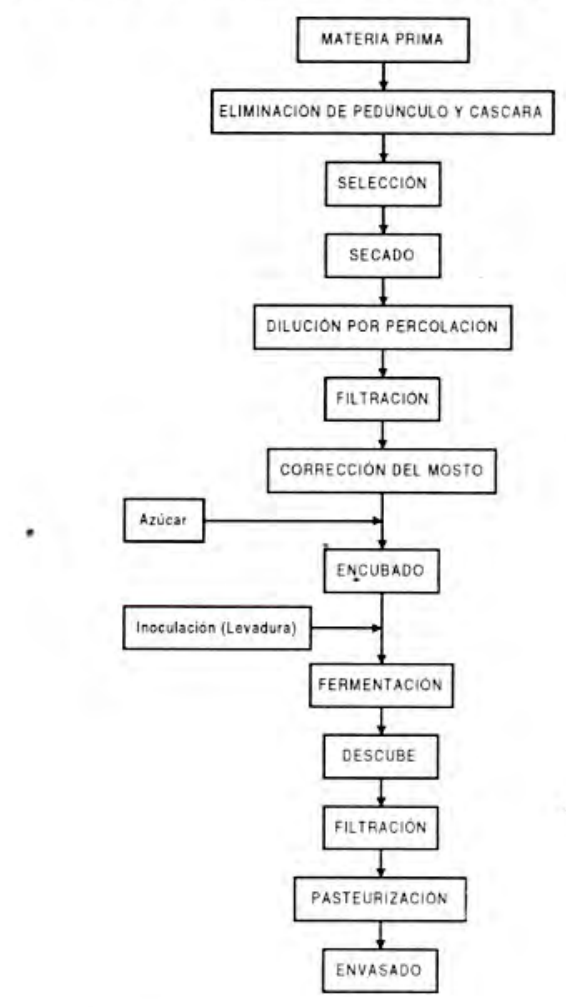

Flujograma 2. Elaboración de Vinagre de Molle.

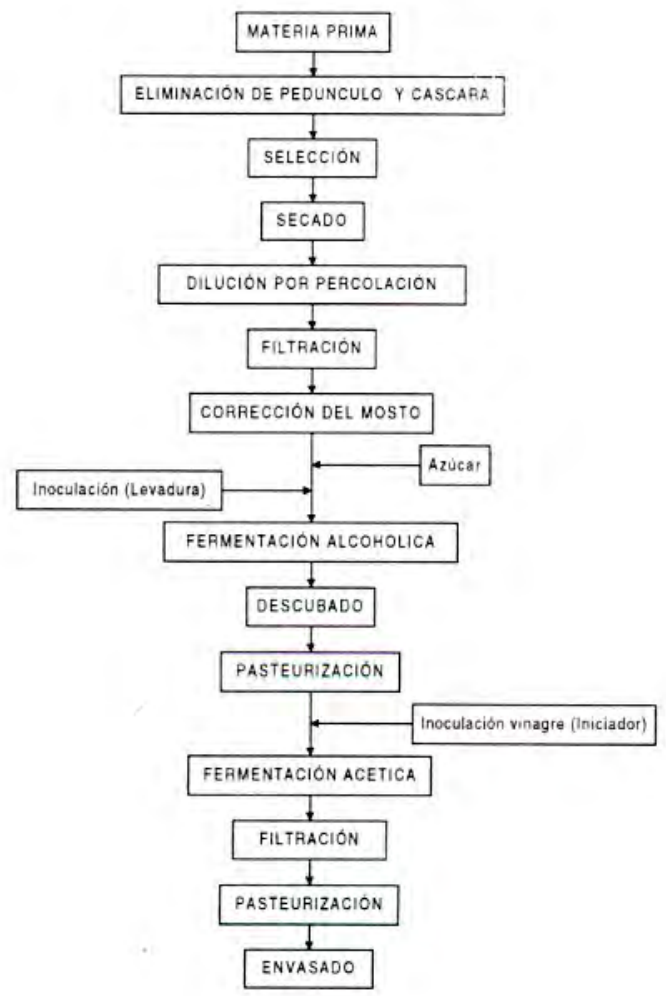




\section{Obtención de Alcohol Etílico}

Se prepararon dos muestras; los frutos de molle maduros y verdes, por separado, seleccionados y limpios fueron secados a 60 y $80^{\circ} \mathrm{C}$, respectivamente, el primero por tres días y el segundo por cuatro días. Se percolaron con agua potable caliente a $85^{\circ} \mathrm{C}$ por 30 minutos. Añadiendo levadura, fueron fermentados, fue necesario corregir el mosto de los frutos verdes. Diariamente se controló el consumo de los sólidos solubles y la acidez hasta lograr su estabilización que ocurrió a las 7 y 11 días, respectivamente. Las muestras se destilaron por separado, habiéndose obtenido alcohol con $13,0 \%$ y $10,0 \%$ de grado alcohólico con frutos maduros y verdes, respectivamente. Sometiendo a sucesivas rectificaciones se llegó a obtener alcohol hasta con 90,4\% de pureza.

\section{Obtención de Aceite Esencial}

Los frutos de molle procedentes de la percolación de la obtención de chicha, vinagre y alcohol, fueron secados a temperatura ambiente por una semana. Para la extracción de aceite se lo dividió en dos partes; una muestra seca sin refrigerar y la otra se refrigeró por cinco días a $5^{\circ} \mathrm{C}$. La extracción de aceite crudo se realizó en un extractor Sohxlet con muestras molidas empleando como solvente hexano, la miscela obtenida se filtró y se sometió a evaporación a $70^{\circ} \mathrm{C}$ por 30 minutos, se dejó a temperatura ambiente y se sometió a destilación por arrastre con vapor de agua, habiéndose obtenido como destilado el aceite esencial al que se añadió sulfato de sodio anhidro a fin de eliminar la humedad residual y se almacenó en un frasco a baja temperatura de 4 a $5^{\circ} \mathrm{C}$ para los análisis correspondientes.

\section{RESULTADOS Y DISCUSIÓN}

Los dos tipos de chicha tienen un color ambar claro brilloso con un aroma agradable y sabor semidulce exquisito. La chicha cuyo mosto se corrigió añadiendo azúcar antes de la fermentación alcanzó un grado alcohólico de $6 \%$ y una acidez de 0,35; mientras que el otro tipo al que no se añadió azúcar llegó a $4 \%$ de grado alcohólico y 0,37 de acidez; resultados que certifican que, ambos tipos de chicha son recomendables para el consumo humano por sus bajos contenidos de acidez, en comparación, por ejemplo, con la chicha de jora que tiene $1,23 \%$ y el vino de mandarina $0,74 \%$ (Bush 1952), además por sus bajos contenidos en alcohol y extracto seco, de
Flujograma 3. Obtención de Alcohol Etilico.

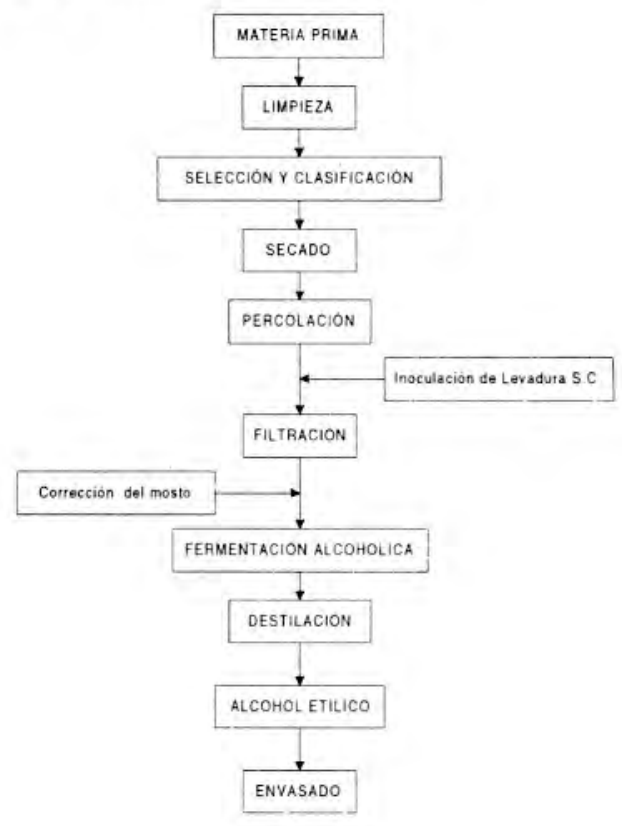

Flujograma 4. Extracción de aceite esencial del fruto de molle.

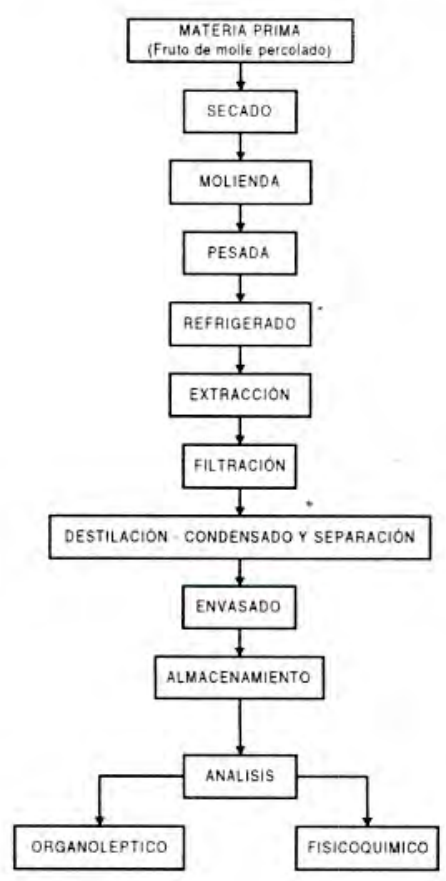


manera que se puede catalogar como un nutriente energético, aunque en su metabolismo no se forman intermediarios que sean aprovechados por la célula, por lo que se puede considerar como generador de calorías.

En cuanto al vinagre, los análisis se realizaron cada semana de fermentación acética, observándose que a la cuarta semana ya presentó una acidez y el $\mathrm{pH}$ aceptables de 4,07 y 2,95 respectivamente, de modo que no fue necesario corregir su acidez, como lo es usualmente en la fabricación de vinagre con materias primas de otras fuentes en los que se añade ácido cítrico; en nuestro caso, simplemente añadimos mosto alcohólico original para luego someter a más tiempo de fermentación, de manera que a la séptima semana se logró obtener un vinagre de buena calidad con $5,0 \%$ de acidez y un $\mathrm{pH}$ de 2,70 parámetros exigidos a los vinagres comerciales obtenidos de otras fuentes.

La conversión del mosto alcohólico en vinagre se produce durante la fermentación acética, acelerada por la levadura en presencia de oxígeno, conforme a la siguiente reacción:

$$
\underset{\mathrm{H}_{2} \mathrm{O}, \mathrm{O}_{2}}{\mathrm{CH}_{3} \mathrm{CH}_{2} \mathrm{OH} \underset{\text { Levadura }}{\longrightarrow}} \mathrm{CH}_{3} \mathrm{COOH} . \mathrm{H}_{2} \mathrm{O}
$$

El vinagre nutricionalmente, carece de valor, pero su inclusión a las comidas, principalmente a las ensaladas, las hace más agradables.

El vinagre obtenido del fruto de molle, presenta un color café claro que tiende a guinda, un aroma característico semejante al ácido y un sabor ácido.

Las legislaciones bromatológicas definen al vinagre como el líquido que deriva de almidón o azúcar que sufren dos fermentaciones sucesivas, alcohólica y acética y especifican que no debe tener menos de $4,1 \%$ ni más de $12,3 \%$ de ácido acético (Blanco 1981), nuestros análisis del vinagre de molle se encuentran dentro de estos parámetros, de manera que califican a este producto como uno de los mejores dentro de su género.

Con el nombre genérico de alcohol se conoce al alcohol etílico (nombre común) o etanol (nombre según normas de la Unión Internacional de Química Pura y Aplicada). Alcohol que en nuestro caso lo obtuvimos de la fermentación de los frutos de molle, catalizada por levadura perteneciente al género
Saccharomyces Cereviseae que, actuando mediante una diastaza (zimasa), produjo el desdoblamiento del azúcar contenido en frutos de molle reduciendo una parte para producir alcohol y la otra oxidando hasta dióxido de carbono, habiéndose producido la siguiente reacción fundamental:

$$
\mathrm{C}_{6} \mathrm{H}_{12} \mathrm{O}_{6} \underset{\mathrm{H}_{2} \mathrm{O}}{\stackrel{\text { Levadura }}{\longrightarrow}} 2 \mathrm{CH}_{3} \mathrm{CH}_{2} \mathrm{OH}+2 \mathrm{CO}_{2}
$$

De acuerdo a los análisis, pueden emplearse tanto frutos maduros frescos como aquellos frutos verdes próximos a entrar en maduración, por contener ambos tipos buena cantidad de sólidos solubles (Brix entre 15 a 20), responsables de la producción de alcohol, aunque transcurrido la fermentación alcohólica, los resultados, no fueron los mismos, pues los frutos maduros son los que fermentaron con mayor rapidez tal como sucedió con una de las muestras. Al final de la fermentación, en la muestra con frutos maduros, alcanzó un $13 \%$ de contenido alcohólico, la que por sucesivas rectificaciones se logró elevar hasta $90,4 \%$, alcohol que fue sometido a los análisis a $20^{\circ} \mathrm{C}$ y 712,24 $\mathrm{mmHg}$ (condiciones del laboratorio).

El alcohol del molle con $90.4 \%$ de pureza, es incoloro, de olor agradable, con sabor quemante, soluble en agua. Para una comparación, al mismo tiempo se analizó tanto el alcohol de molle con el porcentaje señalado como el etanol del $95 \%$ de fábrica, en las mismas condiciones de presión y temperatura de trabajo, habiéndose hallado el punto de ebullición para el alcohol de molle entre 79 y $80^{\circ} \mathrm{C}$ muy cercano al del alcohol de comparación, del mismo modo el índice de refracción y la densidad dieron valores bastantes próximos. Resultados que certifican las condiciones óptimas del alcohol de molle y como tal garantizado para su empleo en los laboratorios según su requerimiento y en los diversos usos para los que se requiera.

Los aceites esenciales, son sustancias odoríferas presentes, prácticamente en todos los vegetales, son muy numerosas y están distribuidas en muchas partes distintas del mismo vegetal, en nuestro caso, lo obtuvimos de los frutos de molle.

Los aceites esenciales son mezclas de varias sustancias orgánicas volátiles y algunos materiales cerosos no volátiles. El término "aceite" en su denominación no se refiere a característica química alguna, sino mas bien a que estas sustancias son 
insolubles en agua, pero solubles en solventes no polares.

Químicamente, la mayor parte de casi todos los aceites esenciales consisten en terpenoides (hidrocarburos isoprenoides) y sus derivados oxigenados. Muchos aceites esenciales que se encuentran en la naturaleza son mezclas de una cantidad de hidrocarburos monoterpenoides $\left(\mathrm{C}_{10} \mathrm{H}_{16}\right)$, e hidrocarburos sesquiterpenoides $\left(\mathrm{C}_{15} \mathrm{H}_{24}\right)$, los cuales sirven principalmente como vehículos para los grupos más importantes de compuestos oxigenados (alcohóles, aldehídos, ácidos grasos y ésteres), los cuales aunque presentes en cantidades mucho menores, son generalmente los productores del olor característico del aceite en cuestión. Todos los monoterpenos y sesquiterpenos son volátiles cuando se les destila con vapor (Braverman, 1980).

El aceite esencial extraído de los frutos de molle, es líquido transparente de olor semejante al mango y maracuyá y de sabor característico, con densidad inferior a 0,9 e indice de refracción de 1,47 datos que sugieren un alto porcentaje de hidrocarburos terpénicos o compuestos alifáticos (Domingüez, 1973). Para una comparación, también se analizaron el aceite esencial de orégano y el aceite de oliva. Los indices de refracción y el índice de acidez de molle, fueron ligeramente superiores al del orégano, lo cual es favorable teniendo presente que, estos índices muestran la presencia de ácidos grasos libres y si se tienen presente que, los aceites en general son evaluados por el contenido de estos ácidos.

El indice de éster en el aceite esencial de molle, fue superior al del orégano, señal de que el primero de los aceites estaría conteniendo mayor cantidad de ésteres que le estarían dando el olor agradable semejante al mango y maracuyá siendo por ello muy apreciados en la industria farmacéutica y cosmética.

Antes de extraer el aceite esencial, se extrajo aceite crudo de molle y se analizó junto con el aceite de oliva a modo de comparación. En el aceite de molle, no se halló peróxidos que sí aparecen en el aceite de oliva, lo que nos hace presumir que el aceite de molle contiene un gran porcentaje de tocoferoles, siendo favorable si se tiene presente que los tocoferoles son los antioxidantes naturales de los aceites vegetales. Según Kirk (1962) los aceites vegetales son importantes solamente en lo que respecta a su contenido de tocoferoles que tienen efectos semejantes a la vitamina $\mathrm{E}$. El índice y equivalente de saponificación hallados fueron inferiores al del aceite en comparación, lo que nos permite suponer que el aceite de molle estaría conteniendo ácidos grasos de cadenas más largas, favoreciendo su estado líquido a temperatura ambiente.

Cuadro 1. Características Fisicoquímicas de dos tipos de Chicha de Molle.

\begin{tabular}{|l|c|c|}
\hline \multicolumn{1}{|c|}{ PARAMETRO } & $\begin{array}{c}\text { CHICHA "A" } \\
\text { (Con azúcar) }\end{array}$ & $\begin{array}{c}\text { CHICHA "B" } \\
\text { (Sin azúcar) }\end{array}$ \\
\hline Brix & 12,5 & 10,5 \\
\hline Densidad $\left(20^{\circ} \mathrm{C}\right.$ ) & 1,05 & 1,040 \\
\hline$\%$ Alcohol & 6 & 4 \\
\hline Acidez total & 0,35 & 0,37 \\
\hline pH & 3,84 & 3,35 \\
\hline$\%$ Extracto seco & 12,12 & 10,40 \\
\hline
\end{tabular}

Fuente: Análisis realizado en Laboratorio.

Cuadro 2. Fermentación Acética en la Elaboración de Vinagre

\begin{tabular}{|l|c|c|c|c|c|c|c|c|}
\hline \multirow{2}{*}{$\begin{array}{c}\text { PARA- } \\
\text { METRO }\end{array}$} & \multicolumn{6}{|c|}{ F E R M E N T A C I Ó N (Días) } \\
\cline { 2 - 9 } & $\mathbf{0}$ & $\mathbf{7}$ & $\mathbf{1 4}$ & $\mathbf{2 1}$ & $\mathbf{2 8}$ & $\mathbf{3 5}$ & $\mathbf{4 2}$ & $\mathbf{4 9}$ \\
\hline Brix & 11,5 & 11,5 & 11,5 & 11,5 & 11,5 & 11,5 & 11,5 & 11,5 \\
\hline$\%$ Acidez & 3,5 & 3,32 & 2,57 & 3,97 & 4,07 & 4,27 & 4,8 & 5,0 \\
\hline $\mathrm{pH}$ & 3,5 & 3,45 & 3,25 & 3,05 & 2,95 & 2,80 & 2,75 & 2,7 \\
\hline
\end{tabular}

Fuente: Datos registrados en Laboratorio.

Cuadro 3. Resultados de la Fermentación Alcoholica.

\begin{tabular}{|c|c|c|c|}
\hline $\begin{array}{c}\text { TIEMPO } \\
\text { FERMENTACIÓN } \\
\text { (Días) }\end{array}$ & $\mathbf{p H}$ & Brix & $\%$ Acidez \\
\hline 1 & 4,0 & 15,0 & 0,11 \\
\hline 2 & 3,9 & 14,0 & 0,33 \\
\hline 3 & 3,8 & 10,0 & 0,35 \\
\hline 4 & 3,6 & 6,5 & 0,36 \\
\hline 5 & 3,4 & 6,0 & 0,38 \\
\hline 6 & 3,3 & 5,2 & 0,39 \\
\hline 7 & 3,3 & 5,0 & 0,39 \\
\hline
\end{tabular}

Fuente: Datos registrados durante la obtención de alcohol etílico. 
Cuadro 4. Características del Alcohol de Molle del 90,4\% (Presión: 712,24 $\mathrm{mmHg}$ ).

\begin{tabular}{|l|c|}
\hline \multicolumn{1}{|c|}{ PARAMETRO } & VALOR OBTENIDO \\
\hline Punto de ebullición & $79-80^{\circ} \mathrm{C}$ \\
\hline Indice de refracción & $1,3655-13658$ \\
\hline Densidad $\left(15^{\circ} \mathrm{C}\right)$ & 0,7939 \\
\hline
\end{tabular}

Cuadro 5. Análisis Fisicoquímico Comparativo de Aceite Esencial.

\begin{tabular}{|l|c|c|}
\hline \multicolumn{1}{|c|}{ PARAMETRO } & $\begin{array}{c}\text { ACEITE } \\
\text { ESENCIAL DE } \\
\text { MOLLE }\end{array}$ & $\begin{array}{c}\text { ACEITE } \\
\text { ESENCIAL DE } \\
\text { OREGANO }\end{array}$ \\
\hline Densidad relativa & 0,70108 & $0,8938-0,8971$ \\
\hline Indice de refracción & 1,4755 & $1,474-1,4752$ \\
\hline Indice de acidez (\%) & 1,104 & 1,000 \\
\hline Indice de éster & 13,04 & 7,13 \\
\hline
\end{tabular}

Fuente: Análisis realizado en Laboratorio.

Cuadro 6. Análisis Fisicoquímico Comparativo de Aceite Crudo.

\begin{tabular}{|l|c|c|}
\hline \multicolumn{1}{|c|}{ PARAMETRO } & $\begin{array}{c}\text { ACEITE CRUDO } \\
\text { DE MOLLE }\end{array}$ & $\begin{array}{c}\text { ACEITE DE } \\
\text { OLIVA }\end{array}$ \\
\hline $\begin{array}{l}\text { Indice de peróxidos } \\
\text { (meq/Kg) }\end{array}$ & 0,00 & max.20 meq/Kg. \\
\hline $\begin{array}{l}\text { Indice de } \\
\text { saponificación (\%) }\end{array}$ & 107 & $187-196$ \\
\hline $\begin{array}{l}\text { Equivalente de } \\
\text { saponificación }\end{array}$ & 157 & $898-857$ \\
\hline
\end{tabular}

Fuente: Análisis realizado en Laboratorio.
Cuadro 7. Análisis Organoléptico de: Chicha, Vinagre, Alcohol y Aceite Esencial.

\begin{tabular}{|c|c|}
\hline CARACT. & OBSERVACIONES \\
\hline $\begin{array}{l}\text { CHICHA } \\
\text { - Color } \\
\text { - Olor } \\
\text { - Sabor }\end{array}$ & $\begin{array}{l}\text { Ambar claro brilloso } \\
\text { Aroma agradable caracteristico } \\
\text { Semi-dulce agradable }\end{array}$ \\
\hline $\begin{array}{l}\text { VINAGRE } \\
\text { - Color } \\
\text { - Olor } \\
\text { - Sabor }\end{array}$ & $\begin{array}{l}\text { Café claro que tiende a guinda } \\
\text { Característico (semejante al ácido } \\
\text { acético) } \\
\text { Acido, no agradable al paladar }\end{array}$ \\
\hline $\begin{array}{l}\text { ALCOHOL } \\
\text { - Color } \\
\text { - Olor } \\
\text { - Sabor }\end{array}$ & $\begin{array}{l}\text { Incoloro } \\
\text { Característico agradable } \\
\text { Caracteristico quemänte }\end{array}$ \\
\hline $\begin{array}{l}\text { ACEITE } \\
\text { ESENCIAL } \\
\text { - Apariencia } \\
\text { - Color } \\
\text { - Olor } \\
\text { - Sabor }\end{array}$ & $\begin{array}{l}\text { Liquido, limpido y trarispicrente } \\
\text { Transparente } \\
\text { Caracteristico (semejante a } \\
\text { mango y maracuyá) } \\
\text { Caracteristico }\end{array}$ \\
\hline
\end{tabular}

Fuente: Análisis organoléptico realizado por los autores. 


\section{CONCLUSIONES}

- Se logró elaborar "Chicha de Molle" de dos tipos, ambos de muy buena calidad y garantizados para su consumo.

- Se logró elaborar "Vinagre de Molle" de buena calidad con acidez aceptable como uno de los mejores vinagres y como tal garantizado para su empleo en la preparación de alimentos, en especial en las ensaladas.

- Se ha obtenido "alcohol etílico" hasta con $90.4 \%$ de grado alcohólico, constituyendo un alcohol de alta pureza.

- Se logró obtener "aceite esencial" con parámetros bastante semejante al del aceite esencial de orégano, constituyendo una garantía para su empleo.

\section{RECOMENDACIONES}

- La elaboración de chicha y vinagre de molle obtenidos a nivel de laboratorio, pueden ampliarse a escala semiindustrial a fin de producir en mayor volúmen, en especial la chicha por ser una bebida refrescante y medicinal (antidiarreica, como tal recomendable para combatir el mal del cólera) que bien puede competir con las bebidas gaseosas de fantasía para aplacar la sed o como un aperitivo durante el almuerzo o después de consumir cualquier tipo de comida.

- Realizar un análisis fisicoquímico de la materia residual de los productos obtenidos para su posible utilización como aditivo en el preparado de alimento para animales.

- Investigarla resinadelmolleparasuposibleempleo en la elaboración de lociones, perfumes, talcos, etc.

- Se incentive a la plantación de árboles de molle en toda la periferie de la ciudad con fines ornamentales y pulmón oxigenador y al mismo tiempo se puedan aprovechar sus frutos en la obtención de productos tan valiosos como los preparados en el presente trabajo.

\section{REFERENCIAS BIBLIOGRÁFICAS}

BERNARDINI, E. (1981). Tecnologia de aceites y grasas. Ed. Alhambra, S.A. España.

BLANCO, T. (1981). Nutrición Humana, Tomo II., LimaPerú

BRAVERMAN, J.B.S. (1980). Introducción a la bioquímica de los alimentos, Ed. El Manual Moderno, S.A: México.

BUSH, C. (1952). Consideraciones médicas sociales sobre la chicha. Excelsior №127 Lima - Perú

DOMINGUEZ, X. (1973). Métodos de investigación Fitoquímica, Ed. Limusa, S.A. México.

EGAN-KIRK-SAWYER (1991). Análisis Químico de Alimentos de Pearson, Ed. CECSA. México.
HISCOX-HOPKINS (1979). EI Recetario Industria!. Ed. Gustavo Gili, S.A. Barcelona - España.

ITINTEC. Normas de aceites y grasas: 209.003 al 209.128 y de bebidas alcohólicas: 210.011 al 210.017 .

KIRK, R.E. (1962). Enciclopedia de Tecnologia Química. Ed. Hispanoamericana. Tomo VIII, México.

MEHLENBACHER, V.C. (1970). Análisis de grasas y aceites Vol. 6 Ed. Umo. Bilbao - España.

SOLOMONS, T.W.S. (1979). Química Orgánica, Ed. Limusa S.A. México.

TORRES, J.M. (1975). Elaboración de Chicha de Molle, Tesis UNA - Lima. 